\title{
Perfil sociodemográfico dos pacientes em cuidados paliativos em um hospital de referência em oncologia do estado do Pará, Brasil*
}

\section{Sociodemographic profile of patients in palliative care at an oncology referral hospital in Pará State, Brazil}

Bárbara Rafaela Bastos', Adrya Karolinne da Silva Pereira', Cinthia Costa de Castro², Maria Margarida Costa de Carvalho ${ }^{3}$

' Universidade do Estado do Pará, Belém, Pará, Brasil

2 Hospital Universitário João de Barros Barreto, Belém, Pará, Brasil

${ }^{3}$ Hospital Ophir Loyola, Belém, Pará, Brasil

\begin{abstract}
RESUMO
OBJETIVO: Descrever o perfil sociodemográfico dos pacientes em cuidados paliativos em um hospital de referência em oncologia do estado do Pará, Brasil. MATERIAIS E MÉTODOS: Estudo retrospectivo, descritivo e transversal, realizado por meio da análise de 239 prontuários de pacientes internados no período de 2010 a 2014 , na Clínica de Cuidados Paliativos Oncológicos do Hospital Ophir Loyola, referência em oncologia no Pará. RESULTADOS: A maioria dos indivíduos era do sexo feminino (59,41\%), com mais de 60 anos de idade $(38,91 \%)$, provenientes da Mesorregião Metropolitana de Belém (46,44\%), com profissão/ocupação "dona de casa" (27,62\%), casados ou em união estável (51,05\%), de religião católica (34,73\%), e com baixos níveis de escolaridade, tendo 38,91\% apenas o ensino fundamental incompleto. Houve predominância de pacientes com histórico de tabagismo (56,90\%) e etilismo $(53,56 \%)$, sem antecedentes familiares de câncer $(41,00 \%)$, internados por situações de urgência/emergência (93,72\%). CONCLUSÃO: Definir o perfil desses pacientes permite uma melhor compreensão das características desses indivíduos, auxiliando a equipe multidisciplinar da clínica de cuidados paliativos no planejamento da assistência prestada, além de contribuir significativamente para a implementação de políticas preventivas de saúde no Estado.
\end{abstract}

Palavras-chave: Perfil de Saúde; Oncologia; Cuidados Paliativos.

\begin{abstract}
OBJECTIVE: To describe the sociodemographic profile of patients in palliative care at an oncology referral hospital in Pará State, Brazil. MATERIALS AND METHODS: A retrospective, descriptive, and cross-sectional study was carried out by analyzing 239 medical records of inpatients at the Oncology Palliative Care Clinic of Hospital Ophir Loyola from 2010 to 2014. RESULTS: The majority of the patients were females (59.41\%), over 60 years of age (38.91\%), from the Metropolitan Region of Belém (46.44\%), with "housewife" occupation (27.62\%), married or in a stable union (51.05\%), Catholic religion (34.73\%), and low levels of schooling (38.91\% of them had incomplete elementary school). There was a prevalence of patients with history of smoking (56.90\%) and alcohol consumption (53.56\%), with no family history of cancer (41.00\%), and hospitalization due to emergency situations (93.72\%). CONCLUSION: The profile of these patients allows a better understanding of their characteristics, helping the multidisciplinary team of palliative care clinic in assistance planning, as well as contributing significantly to preventive health policies be implemented in Pará.
\end{abstract}

Keywords: Health Profile; Oncology; Palliative Care.

\footnotetext{
* Artigo resultado de trabalho de conclusão de curso, apresentado por Bárbara Rafaela Bastos ao Programa de Residências Multiprofissionais em Saúde, área de Oncologia - Cuidados Paliativos, da Universidade do Estado do Pará, em 26 de janeiro de 2017, em Belém, estado do Pará, Brasil.
}

\author{
Correspondência / Correspondence: \\ Bárbara Rafaela Bastos \\ Trav. Barão do Triunfo, 2930B. Bairro: Marco. CEP: 66095-050 - Belém, Pará, Brasil - Tel.: + 55 (91) 98191 - 1404 \\ E-mail: barbararafaela1@gmail.com
}




\section{INTRODUÇÃO}

câncer é uma doença crônico-degenerativa, caracterizada pelo crescimento descontrolado e desordenado de células que impedem o funcionamento normal do organismo; é de etiologia multifatorial, desencadeada principalmente por alterações genéticas, fatores ambientais e estilos de vida'. Atualmente, o câncer é um problema de contexto mundial que vem ganhando cada vez mais atenção devido a eventos variados, como o envelhecimento populacional e fatores próprios do mundo contemporâneo, a exemplo de alimentação e estilo de vida inadequados ${ }^{2}$. No Brasil, estimou-se a ocorrência de 596.070 novos casos de câncer entre 2016 e $2017^{3}$.

As possibilidades de cura do câncer geralmente estão relacionadas ao tempo e ao estágio da doença, ou seja, quanto mais precoce o diagnóstico, mais chances do tratamento promover a cura ${ }^{4}$. Como nem sempre esse diagnóstico é feito precocemente, a doença acaba sendo descoberta em estágio avançado, com chance reduzida e até mesmo sem possibilidade de cura, o que torna essencial a inserção de cuidados paliativos, que objetivam amenizar sinais e sintomas e são realizados por uma equipe multidisciplinar para promover o bem-estar do paciente ${ }^{5}$.

Segundo a Organização Mundial da Saúde, - cuidado paliativo é um tipo de abordagem que objetiva proporcionar qualidade de vida não só aos pacientes, mas também aos familiares, diante de problemas associados a doenças que ameacem a vida, promovendo a prevenção e o alívio do sofrimento, por meio de identificação precoce, avaliação correta, tratamento da dor e de outros problemas de ordem física, psicossocial e espiritual ${ }^{5}$.

O Hospital Ophir Loyola (HOL) é pioneiro na implantação dos cuidados paliativos oncológicos no estado do Pará, oferecendo esse serviço há mais de uma década aos pacientes e seus familiares ${ }^{6}$. Caracterizar o perfil desses pacientes é importante, porque permite conhecer a população que tem vivenciado o câncer avançado e a terapia paliativa no Estado. Esses dados são subsídios para a implantação de ações de promoção, prevenção, diagnóstico e tratamento precoce da doença.

Portanto, este estudo objetivou descrever o perfil sociodemográfico dos pacientes em cuidados paliativos no $\mathrm{HOL}$, referência em oncologia no Pará, Brasil.

\section{MATERIAIS E MÉTODOS}

Trata-se de um estudo retrospectivo, descritivo e transversal, realizado de abril a novembro de 2016, utilizando dados dos prontuários de pacientes oncológicos admitidos entre janeiro de 2010 e dezembro de 2014, na Clínica de Cuidados Paliativos Oncológicos do HOL. De acordo com a Divisão de Arquivo Médico e Estatístico do $\mathrm{HOL}, 627$ pacientes foram admitidos na referida Clínica nesse período. Após a realização do cálculo amostral, com nível de confiança de $95 \%$ e erro amostral preditivo de 5\%, chegou-se ao quantitativo de 239 prontuários a serem analisados.
Os dados dos prontuários foram inseridos em formulário pré-estruturado, com 11 questões fechadas sobre aspectos sociodemográficos (sexo, faixa etária, naturalidade, procedência, escolaridade, ocupação, estado civil, religião, tabagismo, etilismo e antecedentes familiares).

A análise estatística foi realizada utilizando-se métodos descritivos, inferenciais e teste qui-quadrado. As variáveis com valor $\mathrm{p} \leq 0,05$ foram consideradas estatisticamente significantes. Para a edição e formatação das tabelas e gráficos, foram utilizados os programas Microsoft Excel e Microsoft Word 2007; e, para a análise estatística, foi usado o BioEstat v5.3.

Todos os dados obtidos nesta pesquisa foram analisados respeitando-se as normas de pesquisa envolvendo seres humanos, contidas na Resolução No 466/2012 do Conselho Nacional de Saúde7. Este estudo foi aprovado pelo Comitê de Ética em Pesquisa do $\mathrm{HOL}$ em 7 de março de 2016, sob o parecer $\mathrm{n}^{\circ} 1.440 .565$.

\section{RESULTADOS}

Dos 239 prontuários analisados, houve predominância de pacientes do sexo feminino $(59,41 \%)$. Quanto à distribuição por idade, predominou a faixa etária de maiores de 60 anos $(38,91 \%)$. Em relação à proveniência, observou-se que 46,44\% dos pacientes eram naturais da Mesorregião Metropolitana de Belém e 93,72\% internaram após atendimentos de urgência/emergência na triagem do $\mathrm{HOL}$ (Tabela 1).

Observaram-se baixos níveis de escolaridade, visto que $38,91 \%$ dos pacientes tinham apenas o ensino fundamental incompleto. Sobre a ocupação/profissão, $27,62 \%$ afirmaram ser "donas de casa". Quanto ao estado civil, $51,05 \%$ eram casados ou viviam em união estável. Sobre a religião, 34,73\% dos pacientes declararam ser católicos (Tabela 1).

Em relação à dependência química, 56,90\% possuíam algum histórico de tabagismo e 53,56\%, de etilismo. Observou-se ainda que 41,00\% declararam não possuir antecedentes familiares de neoplasias malignas (Tabela 1).

\section{DISCUSSÃO}

Neste estudo, foi verificado que a maioria dos pacientes oncológicos em cuidados paliativos no $\mathrm{HOL}$ eram mulheres, resultado semelhante ao encontrado na literatura. Em países em desenvolvimento, como - Brasil, é esperado o predomínio de câncer no sexo feminino, devido aos altos índices de detecção de neoplasias específicas a esse gênero, como o câncer de colo uterino ${ }^{8,9}$. Em países desenvolvidos, a ocorrência de câncer tende a incidir de forma similar em ambos os sexos, devido aos programas de prevenção em massa desenvolvidos por esses países ${ }^{8}$. Souza et al. ${ }^{10}$ afirmaram ainda que a maior concentração do sexo feminino com câncer avançado também pode ser reflexo da população feminina ser maior em determinadas regiões demográficas. 
Tabela 1 -Distribuição das variáveis sociodemográficas avaliadas de pacientes oncológicos em cuidados paliativos atendidos no $\mathrm{HOL}$, em Belém, estado do Pará, Brasil, de 2010 a 2014

\begin{tabular}{|c|c|c|}
\hline \multirow{2}{*}{ Variáveis } & \multicolumn{2}{|c|}{ Frequência } \\
\hline & $N=239$ & $\%$ \\
\hline \multicolumn{3}{|l|}{ Sexo } \\
\hline Feminino & 142 & 59,41 \\
\hline Masculino & 97 & 40,59 \\
\hline \multicolumn{3}{|l|}{ Faixa etária (anos) } \\
\hline 19-28 & 8 & 3,35 \\
\hline 29-39 & 32 & 13,39 \\
\hline $40-50$ & 56 & 23,43 \\
\hline $51-60$ & 50 & 20,92 \\
\hline$>60$ & 93 & 38,91 \\
\hline \multicolumn{3}{|l|}{ Naturalidade } \\
\hline Mesorregião Metropolitana de Belém & 111 & 46,44 \\
\hline Mesorregião do Nordeste Paraense & 75 & 31,38 \\
\hline Mesorregião do Marajó & 24 & 10,04 \\
\hline Mesorregião do Sudeste Paraense & 7 & 2,93 \\
\hline Mesorregião do Baixo Amazonas & 6 & 2,51 \\
\hline Mesorregião do Sudoeste Paraense & 1 & 0,42 \\
\hline Outros Estados & 15 & 6,28 \\
\hline \multicolumn{3}{|l|}{ Procedência } \\
\hline Triagem & 224 & 93,72 \\
\hline Transferência & 8 & 3,35 \\
\hline Domicílio & 7 & 2,93 \\
\hline \multicolumn{3}{|l|}{ Escolaridade } \\
\hline Analfabeto & 38 & 15,90 \\
\hline Ensino fundamental completo & 30 & 12,55 \\
\hline Ensino fundamental incompleto & 93 & 38,91 \\
\hline Ensino médio completo & 32 & 13,39 \\
\hline Ensino médio incompleto & 4 & 1,67 \\
\hline Superior completo & 11 & 4,60 \\
\hline Superior incompleto & 2 & 0,84 \\
\hline Sem informação & 29 & 12,14 \\
\hline \multicolumn{3}{|l|}{ Ocupação } \\
\hline "Dona de casa" & 66 & 27,62 \\
\hline Autônomo & 49 & 20,50 \\
\hline Lavrador/Agricultor/Pescador & 27 & 11,30 \\
\hline Ferreiro/Carpinteiro/Pedreiro/Pintor/Marceneiro & 17 & 7,11 \\
\hline Profissionais de nível médio & 16 & 6,69 \\
\hline Funcionário público/Aposentado & 12 & 5,02 \\
\hline Segurança/Zelador/Vigilante & 8 & 3,35 \\
\hline Profissionais de nível superior & 8 & 3,35 \\
\hline Estivador/Serviços gerais & 3 & 1,25 \\
\hline Sem informação & 33 & 13,81 \\
\hline \multicolumn{3}{|l|}{ Estado civil } \\
\hline Casado/União estável & 122 & 51,05 \\
\hline Solteiro & 62 & 25,94 \\
\hline Viúvo & 26 & 10,88 \\
\hline Divorciado & 10 & 4,18 \\
\hline Sem informação & 19 & 7,95 \\
\hline \multicolumn{3}{|l|}{ Religião } \\
\hline Católico & 83 & 34,73 \\
\hline Evangélico & 73 & 30,54 \\
\hline Espírita & 2 & 0,84 \\
\hline Outras & 11 & 4,60 \\
\hline Sem informação & 70 & 29,29 \\
\hline \multicolumn{3}{|l|}{ Tabagismo } \\
\hline Sim & 136 & 56,90 \\
\hline Não & 82 & 34,31 \\
\hline Sem informação & 21 & 8,79 \\
\hline \multicolumn{3}{|l|}{ Etilismo } \\
\hline Sim & 128 & 53,56 \\
\hline Não & 89 & 37,24 \\
\hline Sem informação & 22 & 9,20 \\
\hline \multicolumn{3}{|l|}{ Antecedentes familiares } \\
\hline Sim & 95 & 39,75 \\
\hline Não & 98 & 41,00 \\
\hline Sem informação & 46 & 19,25 \\
\hline
\end{tabular}


A faixa etária mais frequente no período analisado foi de > 60 anos. Em um estudo realizado por Ciałkowska-Rysz et al. ${ }^{11}$, também houve predominância de pacientes nessa faixa etária, fato que estaria relacionado ao aumento da expectativa de vida e à queda na taxa de natalidade, ou seja, predomínio da população de idosos, o que pode resultar no aumento de doenças crônico-degenerativas, como o câncer. Segundo o Instituto Brasileiro de Geografia e Estatística ${ }^{12}$, em 2030, o Brasil terá mais idosos do que crianças e jovens, e a mortalidade por câncer terá crescido 45\%. Há uma diminuição progressiva na capacidade de regeneração e divisão celular em idosos e muitos apresentam alguma comorbidade além da neoplasia maligna, o que pode influenciar diretamente no prognóstico da doença e, assim, levá-los à necessidade de cuidados paliativos oncológicos ${ }^{13}$.

Quanto à região de procedência dos pacientes analisados neste estudo, observou-se que 46,44\% residiam na Mesorregião Metropolitana de Belém. A alta incidência nas regiões metropolitanas se deve provavelmente ao estilo de vida adotado nessas regiões. As alterações feitas no meio ambiente, a dieta e o estilo de vida das pessoas podem determinar - surgimento de câncer nas populações e mostram ainda que a exposição aos agentes de polvição estão diretamente ligados ao desenvolvimento de neoplasias malignas, as quais, se não diagnosticadas precocemente, podem se apresentar como uma doença já sem possibilidade de cura ${ }^{14,15}$.

A maioria dos pacientes era oriunda de triagem/emergência. Mierendorf e Gidvani ${ }^{16}$ evidenciaram que pessoas com doenças crônicas, principalmente aquelas com câncer em estágio avançado, necessitam frequentemente do serviço de emergência. Gulini et al. ${ }^{17}$ relataram que, para muitos profissionais, principalmente os que trabalham nos serviços de urgência/emergência, é difícil saber quando determinados procedimentos devem ser mantidos ou suspensos. Para Lourençato et al. ${ }^{18}$, a falta de preparo nas situações de urgência/emergência pode submeter o paciente em cuidado paliativo a tratamentos desproporcionais a sua condição de saúde, gerando sofrimento a ele, a seus familiares e à própria equipe assistencial. Diante desse quadro, observa-se a necessidade de uma equipe multidisciplinar treinada e qualificada para esse tipo de serviço. Além disso, vê-se a importância da elaboração de protocolos que auxiliem e facilitem a tomada de decisões em relação a quais tratamentos devem ou não ser implementados ${ }^{17,18}$.

Quanto à escolaridade, a maioria dos pacientes apresentava apenas o ensino fundamental incompleto. Estudos realizados por Noce e Rebelo ${ }^{19}$ e por Schneider e d'Orsi ${ }^{20}$ mostraram a relação entre aspectos socioeconômicos e o diagnóstico feito quando não existe mais possibilidade de cura, o que pode ser resultante da dificuldade de acesso aos programas preventivos e assistenciais de saúde. A baixa escolaridade também está associada a piores padrões de cuidados à saúde, levando a uma maior possibilidade de sofrimento e uma pior qualidade de vida na fase de finitude ${ }^{19,20}$.

Rushton et al. ${ }^{21}$ destacaram que muitos trabalhadores podem ser expostos a vários carcinógenos durante suas atividades laborativas. Neste estudo, a ocupação predominante foi a de "dona de casa"; entretanto, essa variável não pôde ser associada como um fator de risco para o câncer e nem para o paciente se encontrar em cuidados paliativos.

Foi identificado que $51,05 \%$ dos pacientes pesquisados eram casados ou viviam em união estável, o que mostrou que a associação entre estado civil e paciente em cuidados paliativos não é significativa. Dugno et al. ${ }^{22}$ também não conseguiram estabelecer relação entre o estado civil e a ocorrência de câncer avançado e sem possibilidade de cura em um estudo conduzido no sul do Brasil. No entanto, outros estudos demonstraram que pacientes com câncer e que vivem em união estável têm mais chances de sobrevivência do que aqueles que vivem só $^{21,22}$.

No presente estudo, a maioria declarou algum tipo de religião, tendo havido predominância de pacientes que se declararam católicos. Mesquita et al. ${ }^{23}$, em um estudo sobre a utilização do enfrentamento espiritual por pacientes com câncer, mostraram que mais de $90 \%$ dos mesmos consideravam a religião muito importante. King et al. ${ }^{24}$, analisando as crenças espirituais na fase final de vida, verificaram que houve tendência a diminuir a prescrição analgésica em pacientes com crenças espirituais, indicando que essas estão ligadas a menores níveis de ansiedade e depressão.

Sobre a dependência química, houve predominância de pacientes com histórico de tabagismo $(56,90 \%)$. Park et al. ${ }^{25}$ verificaram que o tabagismo foi responsável por $32,9 \%$ das mortes por câncer em homens adultos na Coreia, e que aproximadamente uma em cada três mortes por câncer seria potencialmente evitável pelo controle adequado do tabagismo. Em relação ao etilismo, $53,56 \%$ dos pacientes apresentaram histórico de consumo de bebidas alcoólicas. Segundo Connor ${ }^{26}$, o consumo de álcool pode estar relacionado à ocorrência de câncer em cerca de sete regiões distintas: orofaringe, laringe, esôfago, fígado, colón, reto e mama feminina. Esse autor relata ainda que o consumo excessivo é o maior problema, embora - baixo consumo também possa predispor ao risco de ocorrência da doença. Wünsch Filho ${ }^{27}$ afirmou que cerca de $4 \%$ dos óbitos em todo o mundo são atribuídos ao consumo de bebidas alcoólicas, e mais da metade desse valor tem ligação com doenças crônicas não transmissíveis, como o câncer.

Quanto aos antecedentes familiares de câncer, a maioria dos pacientes investigados não apresentou esse fator e, por isso, não foi possível fazer associação entre genética e o câncer avançado sem possibilidade de cura. Palmero ${ }^{28}$, avaliando a relação entre hereditariedade e câncer de mama, afirmou que famílias que apresentam muitos casos de câncer, neoplasias bilaterais de mama ou tumores descobertos 
em indivíduos muito jovens deveriam ser avaliadas cuidadosa e rigorosamente, visto que esses fatores são indicadores importantes de risco para o câncer hereditário.

\section{CONCLUSÃO}

Conhecer o perfil desses pacientes contribui para a compreensão das peculiaridades desses indivíduos, auxiliando a equipe multidisciplinar da clínica de cuidados paliativos no planejamento da assistência prestada. Ao se delinear e sugerir intervenções aos pacientes em cuidados paliativos, é fundamental que se considere os recursos, a linguagem utilizada na orientação dos cuidados e o tipo de população mais afetada pela doença. Além disso, pode ajudar o poder público na implementação de campanhas e ações de promoção, prevenção, diagnóstico e tratamento precoce, na tentativa de modificar positivamente o cenário da estimativa do câncer avançado e sem possibilidade de cura no Pará.

\section{CONFLITOS DE INTERESSE}

As autoras declararam não ter havido conflitos de interesse em relação ao estudo.

\section{CONTRIBUIÇÃO DOS AUTORES}

Todas as autoras participaram igualmente das etapas de elaboração do artigo e declaram-se responsáveis pelo seu conteúdo integral, garantindo sua precisão e integridade.

\section{REFERÊNCIAS}

1 Instituto Nacional de Câncer. ABC do câncer: abordagens básicas para o controle do câncer. Rio de Janeiro: INCA; 2011.

2 Teixeira LA, Fonseca CMO. De doença desconhecida a problema de saúde pública: - INCA e o controle do câncer no Brasil. Rio de Janeiro: Ministério da Saúde; 2007.

3 Ministério da Saúde (BR). Instituto Nacional de Câncer "José Alencar Gomes da Silva". Coordenação de Prevenção e Vigilância. Estimativa 2016: incidência de câncer no Brasil. Rio de Janeiro: INCA; 2015.

4 Instituto Oncoguia. Câncer tem cura? [Internet]. 2014 jun [citado 2015 ago 24]. Disponível em: http://www. oncoguia.org.br/conteudo/cancer-tem-cura/81/1/.

5 World Health Organization. WHO definition of palliative care [Internet]. 2012. [cited 2016 Feb 23]. Available from: http://www.who.int/cancer/ palliative/definition/en.

6 Hospital Ophir Loyola. Missão, visão e valores [Internet]. 2013 [citado 2016 set 3]. Disponível em: http://www.ophirloyola.pa.gov.br/institucional/ missao-visao-valores/.

7 Brasil. Ministério da Saúde. Conselho Nacional de Saúde. Resolução $n^{\circ}$ 466, de 12 de dezembro de 2012. Dispõe sobre normas e diretrizes de pesquisas envolvendo seres humanos [Internet]. Brasília: Conselho Nacional de Saúde; 2012 [citado 2016 ago 8]. Disponível em: http://conselho.saude.gov.br/ resolucoes/2012/Reso466.pdf.

8 Rodrigues JSM, Ferreira NMLA. Caracterização do perfil epidemiológico do câncer em uma cidade do interior paulista: conhecer para intervir. Rev Bras Cancerol. 2010 out-dez;56(4):431-41.
9 Sociedade Brasileira de Cancerologia. Câncer ginecológico [Internet]. 2016 out [citado 2016 nov 9]. Disponível em: http://www.sbcancer.org.br/ wpcontent/uploads/2016/10/cancer-ginecologico. pdf.

10 Souza RS, Simão DAS, Lima EDRP. Perfil sociodemográfico e clínico de pacientes atendidos em um serviço ambulatorial de quimioterapia paliativa em Belo Horizonte. REME Rev Min Enferm. 2012 jan-mar; $16(1): 38-47$.

11 Ciałkowska-Rysz A, Kowalczyk M, Gottwald L, Kazmierczak-Łukaszewicz S. The comparison of common cancer types and the coincidence of concomitant chronic diseases between palliative home care patients in Lodz Voivodeship and the general Polish population. Arch Med Sci. 2012 Jul;8(3):496-503.

12 Instituto Brasileiro de Geografia e Estatística. Sinopse do censo demográfico [Internet]. Brasília: IBGE; 2010 [citado 2016 nov 6]. Disponível em: http://www.censo2010.ibge.gov.br/.

13 Antunes YPPV, Bugano DDG, Giglio A, Kaliks RA, Karnakis T, Pontes LB. Características clínicas e de sobrevida global em pacientes oncológicos idosos num centro oncológico terciário. Einstein. 2015 out-dez; 13(4):487-91.

14 Loomis D, Huang W, Chen G. The International Agency for Research on Cancer (IARC) evaluation of the carcinogenicity of outdoor air pollution: focus on China. Chin J Cancer. 2014 Apr;33(4):189-96.

15 Aleksandrova $K$, Pischon $T$, Jenab $M$, Bueno-de-Mesquita HB, Fedirko V, Norat T, et al. Combined impact of healthy lifestyle factors on colorectal cancer: a large European cohort study. BMC Medicine. 2014;12:168.

16 Mierendorf SM, Gidvani V. Palliative care in the emergency department. Perm J. 2014;18(2):77-85. 
17 Gulini JEHMB, Nascimento ERP, Moritz RD, Rosa LM, Silveira NR, Vargas MAO. Intensive care unit team perception of palliative care: the discourse of the collective subject. Rev Esc Enferm USP. 2017 May; $51: e 03221$.

18 Lourençato FM, Santos AFJ, Ficher AMFT, Santos JC, Zoppi D, Giardini MH, et al. Implantação de serviço de cuidados paliativos no setor de emergência de um hospital público universitário. Rev Qual HC. 2016;127-33.

19 Noce CW, Rebelo MS. Avaliação da relação entre tamanho do tumor e características sociais em pacientes com carcinoma de células escamosas bucal. Rev Bras Cancerol. 2008;54(2):123-9.

20 Schneider IJC, d'Orsi E. Sobrevida em cinco anos e fatores prognósticos em mulheres com câncer de mama em Santa Catarina, Brasil. Cad Saude Publica. 2009 jun;25(6): 1285-96.

21 Rushton L, Bagga S, Bevan R, Brown TP, Cherrie JW, Holmes $P$, et al. Occupation and cancer in Britain. Br J Cancer. 2010 Apr; 102(9):1428-37.

22 Dugno MLG, Soldatelli JS, Daltoé T, Rosado JO, Spada P, Formolo F. Perfil do câncer de mama e relação entre fatores de risco e estadiamento clínico em hospital do Sul do Brasil. Rev Bras Oncol Clin. 2014 abr-jun; 10(36):60-6.
23 Mesquita AC, Chaves ECL, Avelino CCV, Nogueira DA, Panzini RG, Carvalho EC. A utilização do enfrentamento religioso/espiritual por pacientes com câncer em tratamento quimioterápico. Rev Latino-Am Enfermagem. 2013 mar-abr;21(2):[07 telas].

24 King $M$, Llewellyn $H$, Leurent B, Owen $F$, Leavey $G$, Tookman A, et al. Spiritual beliefs near the end of life: a prospective cohort study of people with cancer receiving palliative care. Psychooncology. 2013 Nov;22(1 1):2505-12.

25 Park S, Jee SH, Shin HR, Park EH, Shin A, Jung $\mathrm{KW}$, et al. Attributable fraction of tobacco smoking on cancer using population-based nationwide cancer incidence and mortality data in Korea. BMC Cancer. 2014 Jun; 14:406.

26 Connor J. Alcohol consumption as a cause of cancer. Addiction. 2017 Feb; 1 12(2):222-8.

27 Wünsch Filho V. Consumo de bebidas alcoólicas e risco de câncer. Rev USP. 2012 dez;2013 fev; $96: 37-46$.

28 Palmero El. Hereditariedade e câncer de mama. Onco\& [Internet]. 2013 set-out [citado 2014 mar 20];4(19):38-41. Disponível em: http:// studylibpt.com/doc/1555308/hereditariedade-ec\%C3\%A2ncer-de-mama. 